\title{
Acyclovir Unresponsive Herpes Simplex Encephalitis in a child successfully treated with the addition of Foscarnet. Case report
}

\author{
Zeynep G. Gayretli Aydin, M.D. ${ }^{a}$, Gonul Tanir, Assoc. Prof. ${ }^{a}$, Cigdem Genc Sel, M.D. ${ }^{b}$, \\ Yasemin Tasci Yildiz, M.D. ', Turkan Aydin Teke, Assoc. Prof. ${ }^{a}$ and Ayse Kaman, M.D. ${ }^{a}$
}

\begin{abstract}
Herpes simplex encephalitis (HSE) is the most common cause of sporadic focal encephalitis worldwide. Acyclovir is the treatment of choice of HSE since the 1980s. After the widespread use of acyclovir, HSE related mortality rate had reduced but resistant strains emerged. Acyclovir resistant HSV incidence was reported as about $0.5 \%$ and $3.5 \%-10 \%$ in immunocompetent and immunocompromised patients, respectively. Herein, a 12-year-old immunocompetent patient with HSV-1 encephalitis who was successfully treated with combined acyclovir and foscarnet therapy is described. In the case of deteriorating clinical condition under acyclovir treatment even if the absence of demonstration of increased CSF HSV viral load, the possibility of acyclovir resistant HSE and the addition of foscarnet to the acyclovir treatment might be considered.
\end{abstract}

Key words: herpes simplex encephalitis, acyclovir, foscarnet, child.

http: / / dx.doi.org/ 10.5546/ aap.2019.eng.e47

To cite: Gayretli Aydin ZG, Tanir G, Genc Sel C, Tasci Yildiz Y, et al. Acyclovir Unresponsive Herpes Simplex Encephalitis in a child successfully treated with the addition of Foscarnet. Case report. Arch Argent Pediatr 2019;117(1):e47-e51.

\section{INTRODUCTION}

Herpes simplex encephalitis (HSE) is the most common cause of sporadic focal encephalitis worldwide. ${ }^{1,2}$ The incidence ranges from 1 to 2 cases per 500000 population per year. ${ }^{3}$ Acyclovir which is a nucleoside analogue of guanosine is the treatment of choice of HSE. After the use of acyclovir, while HSE related mortality

a Division of Pediatric Infectious Diseases.

b. Division of Pediatric Neurology, Department of Pediatrics.

c. Department of Pediatric Radiology.

Dr. Sami Ulus Maternity and Children's Research and

Education Hospital, Ankara, Turkey.

E-mail address:

Zeynep G. Gayretli Aydin, M.D.: zggayretli@gmail.com

Funding: None.

Conflict of interest: None.

Received: 2-23-2018

Accepted: 9-17-2018 rate had reduced, resistant strains emerged. Acyclovir resistant HSV incidence was reported $0.5 \%$ and $3.5 \%-10 \%$ in immunocompetent and immunocompromised patients respectively. ${ }^{4}$ Herein, a 12-year-old immunocompetent patient with HSV-1 encephalitis who was succesfully treated with combined acyclovir and foscarnet therapy is described.

\section{CASE REPORT}

A 12-year-old boy was admitted to the pediatric emergency department with a 4-day history of fever and headache, 1-day history of irrelevant speech, disorientation and intermittant vomiting. His body temperature $38.5^{\circ} \mathrm{C}$; heart rate was 116 beats per minute. He was lethargic, giving slow and illogical responses to questions. He had neck stiffness with negative Kernig and Brudzinski signs. The remainder neurological examination was normal. Lumbar puncture (LP) was performed that revealed cerebrospinal fluid (CSF) pleocytosis ( $>610$ white blood cells $/ \mathrm{mm}^{3}$ ) with a lymphocytic $(80 \%)$ predominance, CSF protein $93.8 \mathrm{mg} / \mathrm{dl}$ (normal range: $15-45 \mathrm{mg} / \mathrm{dl}$ ) and CSF glucose $85 \mathrm{mg} / \mathrm{dl}$ (simultaneous blood glucose $127 \mathrm{mg} / \mathrm{dl}$ ). On CSF Gram stain, no organisms were seen. Limbic panel from serum and CSF was negative. Empirical treatment of ceftriaxone $(100 \mathrm{mg} / \mathrm{kg} /$ day, intravenously [IV], every 12 hours), clarithromycin ( $15 \mathrm{mg} / \mathrm{kg} /$ day, IV, every 12 hours) and acyclovir (1500 mg/ $\mathrm{m}^{2} /$ day, IV, every 8 hours) was commenced for the possible bacterial meningitis and HSE, respectively. Magnetic resonance imaging (MRI) of the brain at the second day of admission revealed hyperintensity in $\mathrm{T} 2 \mathrm{w}$ images and meningeal contrast enhancement in gyrus rectus of the frontal lobe, hippocampus, amygdala, hypothalamus and mamillar regions of the left cerebral hemisphere. He also had minimal nodular hyperintensity in right temporal lobe in T2w images (Figure 1). First awake and sleep electroencephalography (EEG) records revealed diffuse slowing of background activity in the left temporo-occipital region. On the follow-up, positive CSF HSV-1 polymerase chain reaction 
(PCR), positive serum HSV IgG and negative HSV IgM were detected. CSF HSV-1 PCR was detected by using Real Time Multiplex RT PCR Fast Track Diagnostics Viral Meningitis commercial kit (Fast Track Diagnostics, Luxemburg) and ABI 7500 Real-Time PCR (Applied Biosystems, USA). HSE was diagnosed and treatment with ceftriaxone was discontinued. At the $14^{\text {th }}$ day of acylovir treatment, the patient's neurologic symptoms detoriated, with lethargy, disorientation and behavioral changes. Subsequently vomiting and mild bradycardia without hypertension occurred. Neurologic examination revealed hyperactive deep tendon reflexes of lower limbs. At the next day two tonic convulsions lasting for 40 seconds were observed. Antiepileptic treatment with levetiracetam was initiated. His fundoscopic examination revealed bilateral retinal vascular dilatation and retinal vascular tortuosity with the suspicion of increased intracranial pressure. Second awake and sleep EEG records after the seizures revealed diffuse slowing of background activity with a paroxysmal activity in the right temporal region. Repeat cranial MRI showed increased edema encompassing gyrus rectus of frontal lobe, hippocampus, amygdala, hypothalamus and mamillar regions of the left hemisphere as well as and increased hyperintensity and edema in gyrus rectus of right frontal lobe and temporal region (Figure 2). Second LP for the presence or abscence of HSV1 persistence, could not be performed in this instance. Dexamethasone and $3 \% \mathrm{NaCl}$ were started for cerebral edema. Although it could

FIGURE 1: Transverse T2w image MRI taken on the first day of admission, depicts edema and increased signal intensity at the left temporal and frontal lobes. Similar changes are observed at a small area at the right temporal lobe (1a). No hemorrhage is noted on T1w image (1b). After contrast medium administration meningeal/pial enhancement is observed on the left (1c).
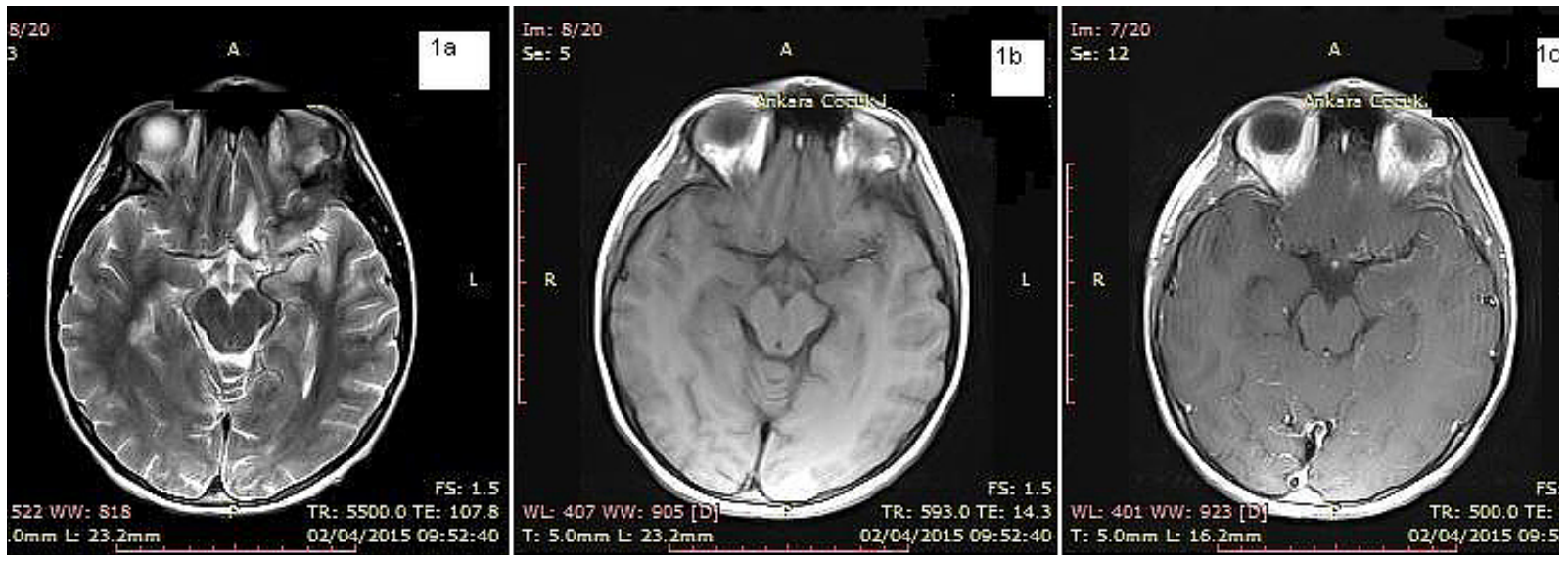

FIGURE 2: T2w image reveals an increase on the edema and the signal intensity of the left temporal and frontal lobes, as well as at the right temporal lobe (2a). On precontrast T1w image, incresed cortical signal is observed consistent with hemorrhage (2b). After contrast medium administration increased meningeal/pial enhancement is observed (2c).
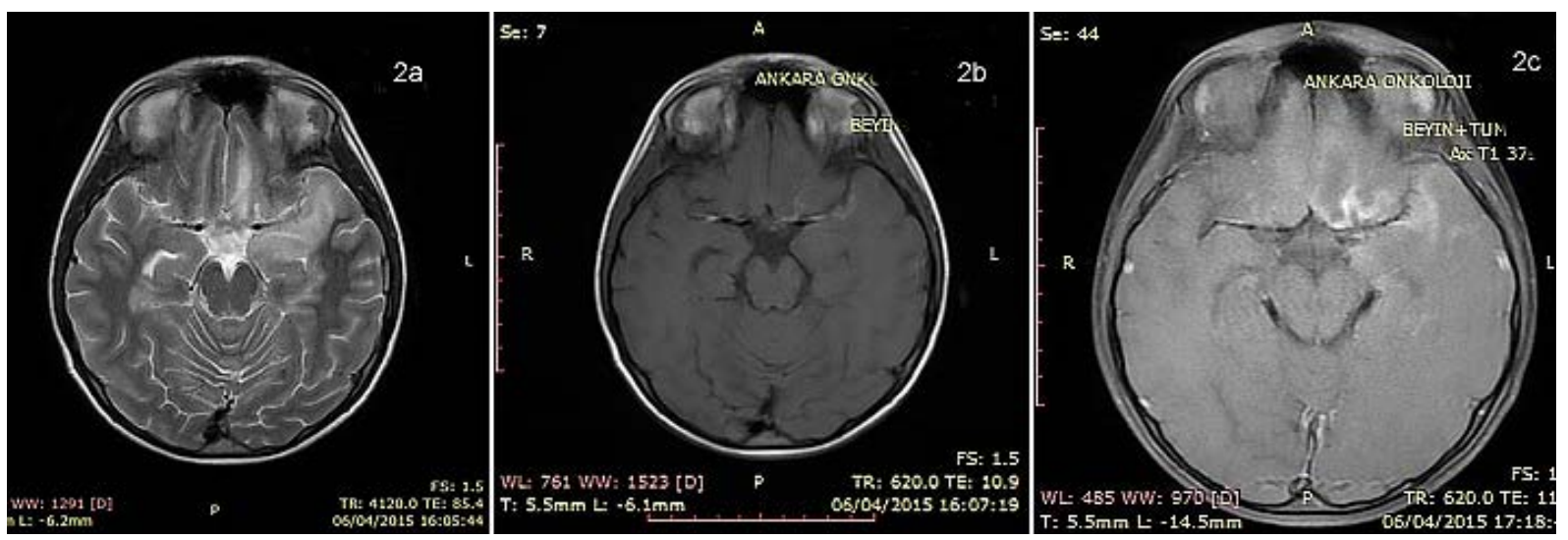
not be possible to confirm the drug resistance by molecular methods, it was thought that this might be a clinical resistance phenomenon to acyclovir. Foscarnet (60 mg/kg, IV, every 12 hours) was added. This therapeutic alteration together with cerebral edema treatment resulted in a dramatic improvement in the patient's overall condition. He became alert and able to adequately answer questions. Fundoscopic examination revealed normal findings. Control LP revealed 20 cells $/ \mathrm{ml}$, normal glucose, protein levels and negative HSV-1 DNA in CSF on day 17 of foscarnet and on day 33 of acyclovir treatment. Acyclovir and foscarnet treatment was stopped with these findings. In the course of the disease, corticosteroid dosage was tapered down. The patient was discharged without neurological sequele. On follow-up, cranial MRI, corticolaminar necrosis in gyrus rectus of frontal lobe, hippocampus, amygdala, hypothalamus and mamillar regions of the left hemisphere in T1w images and decrease in edema and hyperintense signals at the right temporal lobe were detected (Figure 3).

\section{DISCUSSION}

HSV encephalitis is the most common identified cause of viral encephalitis. ${ }^{5}$ Herpes simplex infections of the central nervous system $(\mathrm{CNS})$ are the most severe and destructive infections among all viral infections. ${ }^{3}$ Patients with HSV encephalitis present with fever, altered consciousness, headache, disorientation, seizures, behavior or personality change, memory disturbance, motor deficit and speech disturbances, in order of frequency. ${ }^{5}$ The patient had most of them. Pleocytosis with a lymphocytic predominance, moderately elevated protein and normal glucose levels are the most common CSF findings. MRI and EEG that revealed abnormalities of the temporal lobes are supporting diagnostic tests. ${ }^{3}$ All of the CSF, MRI and EEG findings of our patient were suggestive for HSE, and positive CSF HSV PCR test provided the definitive diagnosis. In HSV encephalitis, it is recommended to repeat $L P$ after 21 days from the initiation of acyclovir treatment. If HSV persists in the CSF, LP must be performed weekly whether HSV in the CSF is present.

Acyclovir is a drug of choice for HSE treatment. Initiation of acyclovir treatment at an earlier stage is known as associated with better prognosis of HSV encephalitis. ${ }^{5}$ Acyclovir acts by affecting two viral enzymes. Acyclovir is phosphorylated by viral thymidine kinase (vTK) to form ACV-mono-phosphate, which is then phosphorylated by cellular kinases and become active form ACV-triphosphate (ACV-TP). ACVTP competitively inhibits viral DNA polymerase. ${ }^{6}$

Mortality rate of HSE was reduced after the introduction of acyclovir. The increasing use of acyclovir may lead to the development of HSV resistance to this drug. Mutations occuring in TK are the most frequent cause (95\%) of acyclovirresistance. ${ }^{7}$ A loss of TK activity, an alteration of TK substrate specificity and an alteration of DNA polymerase activity are responsible for

FIGURE 3: On the $3^{\text {rd }}$ week of admission, this Flair T2w image showes involvement of the girus rectus of the right frontal lobe (3a). T1w image reveals no hemorrhage (3b).
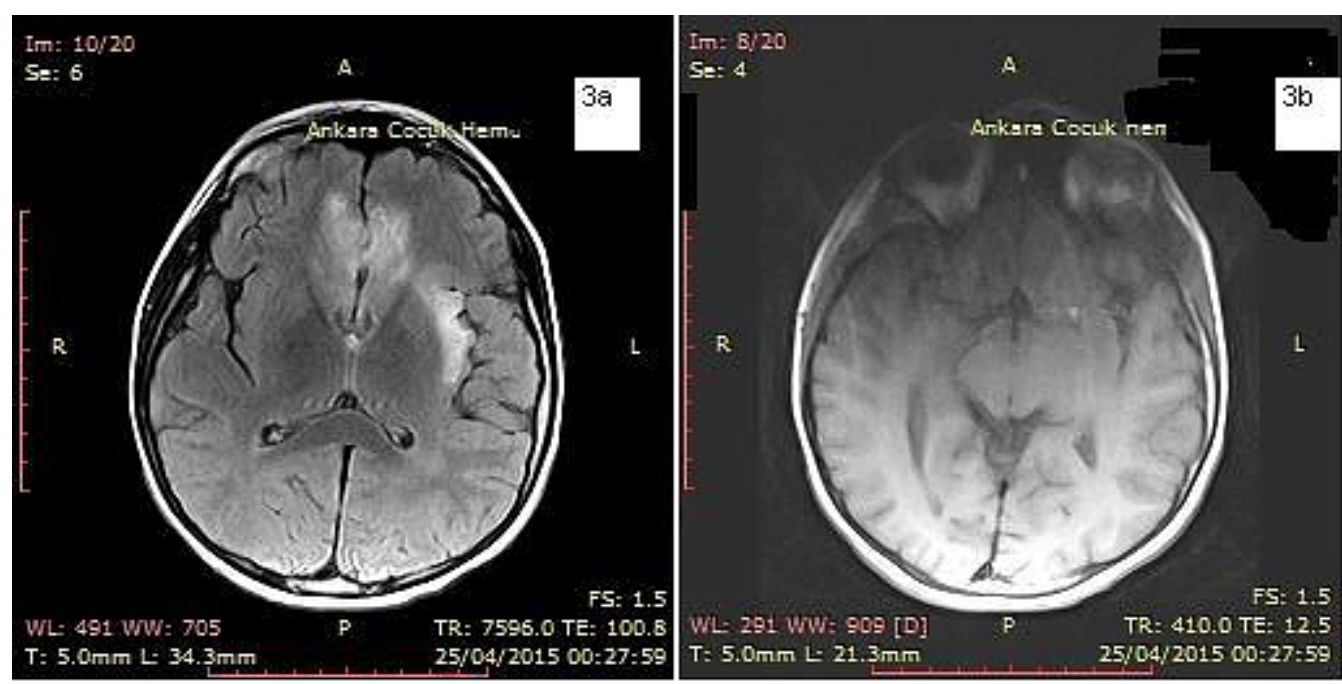
acyclovir resistance. ${ }^{8}$ Resistance to acyclovir has been reported as the range of $3.5 \%-10 \%$ among immunocompromised patients. In a recent study of 1425 patients with HSV infection that aimed to reassess the status of HSV resistance to acyclovir between 2002 and 2011, it was found that the prevalence of resistance in immunocompetent patients did not exceed $0.5 \%$, whereas in immunocompromised patients, a significant increase was observed. ${ }^{4}$ Our patient was a healthy 12-year-old boy. He had deteriorating clinical condition and MRI findings although earlier and recommended dosage of acyclovir treatment. For this reason we suggested the resistance to acylovir. To treat acyclovir resistant infections, drugs affecting in different mechanisms may be used. Foscarnet, which acts directly on viral DNA polymerase, is an alternative choice. There were some case reports of acyclovir-resistant HSV encephalitis who had been treated successfully with the combined acyclovir and foscarnet treatment. A 27-year-old, immunocompetent woman with HSE whose clinical course worsened and HSV-1 load increased under acyclovir treatment, and improved after the combined treatment with foscarnet, had been reported. Five nonsynonymous mutations in nucleotide positions of the thymidine kinase gene (UL23) of the HSV-1 strain had been demonstrated in this case. ${ }^{9}$ Thymidine-kinase mutant virus in a 50-year-old woman with HSE who receiving anti-tumor necrosis factor monoclonal antibodies adalimumab was reported. The combined foscarnet and acyclovir treatment resulted improvement in this case also. ${ }^{10}$ In another case report, a 13-day-old boy with HSV-1 encephalitis while treating with acyclovir, the clinical status became deteriorated and a mutation in the viral thymidine kinase gene (nucleotide G375T, amino acid Q125H) was demonstrated. Vidarabine had been added to the therapeutic regimen from the fifth week of the treatment course because of foscarnet was not available in this case. ${ }^{11}$ A 19 year-old female patient with HSE was reported in a previous study. She was treated with appropriate dosage and duration of acyclovir, but her clinical condition worsened significantly during the following days. Although the acyclovir resistance test can not be done, foscarnet treatment was added to acyclovir therapy. Dramatic clinical improvement was occured after the addition of foscarnet. ${ }^{12}$ Unfortunately we were unable to test increased HSV-1 load and acyclovir resistance. Because of the inability of isolation of the virus from CSF, the sensitivity test of the HSV to acyclovir is very difficult. Although the occurrence of acyclovir resistance in immunocompetent patients is relatively rare, the deterioration of clinical condition and neuroradiological findings over subsequent days despite acyclovir treatment in the present case, indicated possible acyclovir resistance. Beside this, prompt clinical improvement soon after the administration of foscarnet and supportive treatment pointed us to clinical resistance to acyclovir.

We thought that cerebral edema as a part of worsening clinical condition may be developed during the course of HSE encephalitis despite acyclovir treatment. For this reason, control LP could be precluded in the clinical practice. In the case of deteriorating clinical condition under acyclovir treatment even if the absence of demonstration of increased CSF HSV viral load, the possibility of acyclovir resistant HSE and the addition of foscarnet might be considered.

\section{REFERENCES}

1. Stahl JP, Mailles A, De Broucker T. Herpes simplex encephalitis and management of acyclovir in encephalitis patients in France. Epidemiol Infect. 2012; 140(2):372-81.

2. Granerod J, Ambrose HE, Davies NW, Clewley JP, et al. Causes of encephalitis and differences in their clinical presentations in England: a multicentre, population-based prospective study. Lancet Infect Dis. 2010; 10(12):835-44.

3. Whitley RJ, Kimberlin DW. Herpes simplex encephalitis: children and adolescents. Semin Pediatr Infect Dis. 2005; 16(1):17-23.

4. Frobert E, Burrel S, Ducastelle-Lepretre S, Billaud G, et al. Resistance of herpes simplex viruses to acyclovir: an update from a ten-year survey in France. Antiviral Res. 2014; 111:36-41.

5. Schiffer JT, Corey L. Herpes Simplex Virus. In Bennett J, Dolin R, Blaser M (eds). Mandell, Douglas and Bennet principles and practise of infectious disease. 8 ed. Philadelphia: Saunders - Elsevier; 2015.P.1713-30.

6. Wang LX, Takayama-Ito M, Kinoshita-Yamaguchi H, Kakiuchi $S$, et al. Characterization of DNA Polymerase-Associated Acyclovir-Resistant Herpes Simplex Virus Type 1: Mutations, Sensitivity to Antiviral Compounds, Neurovirulence, and In-Vivo Sensitivity to Treatment. Jpn J Infect Dis. 2013; 66(5):404-10.

7. Hill EL, Hunter GA, Ellis MN. In vitro and in vivo characterization of herpes simplex virus clinical isolates recovered from patients infected with human immunodeficiency virus. Antimicrob Agents Chemother. 1991; 35(11):2322-8.

8. Larder BA, Cheng YC, Darby G. Characterization of abnormal thymidine kinases induced by drug-resistant strains of herpes simplex virus type 1. J Gen Virol. 1983; $64 \mathrm{Pt}$ 3:523-32.

9. Schulte EC, Sauerbrei A, Hoffmann D, Zimmer C, et al. Acyclovir resistance in herpes simplex encephalitis. Ann Neurol. 2010; 67(6):830-3.

10. Schepers K, Hernandez A, Andrei G, Gillemot S, et al. Acyclovir-resistant herpes simplex encephalitis in a patient treated with anti-tumor necrosis factor- $\alpha$ monoclonal antibodies. J Clin Virol. 2014; 59(1):67-70. 
11. Kakiuchi S, Nonoyama S, Wakamatsu H, Kogawa K, et al. Neonatal herpes encephalitis caused by a virologically confirmed acyclovir-resistant herpes simplex virus 1 strain. J Clin Microbiol. 2013; 51(1):356-9.
12. Katyal N, Taqui AM, Tepper D, Beary JM, et al. Fulminant Herpes Simplex Virus Type I Encephalitis Despite Maximal Medical Therapy. Cureus. 2018; 10(4):e2467. 